\title{
Laser fabrication of diamond micro-cutting tool-related geometries using a high-numerical aperture micro-scanning system
}

\author{
Gregory Eberle $^{1} \cdot$ Claus Dold $^{2} \cdot$ Konrad Wegener $^{1}$
}

Received: 16 January 2015 / Accepted: 26 April 2015 /Published online: 17 May 2015

(C) Springer-Verlag London 2015

\begin{abstract}
The machining of micro-geometries requires a corresponding micro-cutting tool. Up to now, industry primarily fabricates such tools by grinding or electrical discharge machining. In this paper, an overview of the direct laser fabrication of micro-cutting tool-related geometries on polycrystalline diamond composites and single crystal diamond is presented. This is made possible using picosecond laser pulses operating at second harmonics and a micro-scanning deflection system exhibiting a high-numerical aperture. The generated geometries are inspected using scanning electron microscopy while quality of the cutting edge radius and graphitisation is investigated. The laser ablation process is further enhanced by demonstrating the feasibility of a sequential roughing and finishing strategy.
\end{abstract}

Keywords Micro-cutting tools · Picosecond laser .

Diamond $\cdot$ Raman $\cdot$ Laser ablation $\cdot$ Volume ablation

$\begin{array}{ll}\text { Nomenclature } \\ \varnothing & \text { Diameter [ } \mu \mathrm{m}] \\ \beta & \text { Wedge angle of cutting edge } \\ \lambda & \text { Wavelength [nm] } \\ \tau_{\mathrm{p}} & \text { Pulse duration }[\mathrm{fs}, \mathrm{ps}, \mathrm{ns}]\end{array}$

Gregory Eberle

eberle@iwf.mavt.ethz.ch; http://www.iwf.mavt.ethz.ch

Claus Dold

claus.dold@ewag.com; http://www.ewag.com

Konrad Wegener

wegener@iwf.mavt.ethz.ch; http://www.iwf.mavt.ethz.ch

1 Institute of Machine Tools and Manufacturing (IWF), ETH Zurich, Leonhardstrasse 21, 8092 Zurich, Switzerland

2 EWAG AG, Industriestrasse 4, 4554 Etziken, Switzerland
D4 $\sigma$ Second moment focal spot diameter $[\mu \mathrm{m}]$

$F \quad$ Fluence $\left[\mathrm{J} / \mathrm{cm}^{2}\right]$

$F_{\text {th }} \quad$ Ablation threshold $\left[\mathrm{J} / \mathrm{cm}^{2}\right]$

$f_{\text {eff }} \quad$ Effective focal length of focussing objective [mm]

$f_{\text {rep }}$ Frequency $[\mathrm{kHz}]$

$M^{2} \quad$ Beam quality [-]

$R_{\mathrm{a}} \quad$ Arithmetic mean roughness [nm]

$r_{\text {edge }} \quad$ Cutting edge radius $[\mu \mathrm{m}]$

$t_{\mathrm{f}} \quad$ Finishing time [min]

$t_{\mathrm{r}} \quad$ Roughing time [min]

$T_{\mathrm{g}} \quad$ Graphitisation temperature $\left[{ }^{\circ} \mathrm{C}\right]$

$z_{\text {step }} \quad$ Layer thickness step size of $z$-axis $[\mu \mathrm{m}]$

$v \quad$ Feed rate of laser beam or workpiece $[\mathrm{mm} / \mathrm{s}]$

\section{Introduction}

It is often argued that the laser beam as a tool will replace the functionality of cutting tools. For instance, the laser cutting of reinforced polymers demonstrated by Klotzbach et al. [1] and the laser drilling of holes in metal demonstrated by Ancona et al. [2] are replacing milling and drilling cutting tools, respectively. However, cutting tools exhibit many advantages which the laser beam itself cannot fulfil. For instance, cutting tools are cost-effective, achieve high material removal rates, can perform internal processing (e.g. undercuts and T-slots), can achieve mirror-like surface finishes and mechanical CNC axes can be synchronised to them. Since micro-cutting tools currently exhibit severe limitations, laser technology has been able to overtake many of its applications. This is due to limitations of current micro-cutting tool fabrication techniques such as grinding and electrical discharge machining (EDM). For this reason, an alternative approach is proposed utilising laser technology to enhance the features and quality of microcutting tools made from diamond, enabling such tools to 
perform significantly better and reinforce its associated advantages in machining processes.

\subsection{State of the art}

According to Huo and Cheng [3], commercially available two-fluted micro-milling tools are available in diameters ranging from $\varnothing=100$ to $1000 \mu \mathrm{m}$ while single-fluted or spade type micro-milling tools can exhibit smaller diameters ranging from $25 \leq \emptyset<100 \mu \mathrm{m}$. Micro-drilling tools are commercially available in diameters ranging from $\varnothing=50$ to $300 \mu \mathrm{m}$ while smaller than $\varnothing<50 \mu \mathrm{m}$ are available but of only the spade type geometry. Micro-cutting tools are predominately made from coated tungsten carbide while ultraprecise applications dictate the use of diamond (natural, chemical vapour deposition or monocrystalline). Due to conventional fabrication techniques, diamond micro-cutting tools are simplistic in geometry, exhibit long processing times and are prone to failure during fabrication. Diamond is an outstanding material owing to its high hardness and high thermal conductivity. However, it is a challenging material to process mechanically (e.g. grinding, sawing, polishing) due to its high hardness, to process by electrical discharges due to its low electrical conductivity or to process by laser due to its transparency as well as its low graphitisation onset temperature at atmospheric pressure and given the presence of oxygen. For this reason, the use of ultrashort pulsed laser technology is necessary in order to mitigate thermal influences, achieve efficient absorption, maintain form tolerances, achieve a defined cutting edge and minimise surface roughness. The laser fabrication of diamond cutting tool geometries is investigated over a wide range of pulse durations and wavelengths. Harrison et al. [4] used a $\tau_{\mathrm{p}}=20$ to $200 \mathrm{~ns}$, $\lambda=1064 \mathrm{~nm}$ laser in order to cut polycrystalline diamond (PCD) composites and generate cutting edges. Everson and Molian [5] used a $\tau_{\mathrm{p}}=100 \mathrm{~ns}, \lambda=1064 \mathrm{~nm}$ laser to cut out a PCD composite blank. Here, the topic of surface roughness and topography as well as graphitisation was covered. However, post-processing is necessary when using nanosecond pulse durations to achieve the quality required by industry. For this reason, Dold et al. [6] used a $\tau_{\mathrm{p}}=10 \mathrm{ps}, \lambda=1064 \mathrm{~nm}$ laser and optimised the laser ablation process for PCD composite cutting inserts. The laser processed inserts exhibited no grain breakouts and faster processing times. The performance of the laser processed and ground inserts were evaluated during turning operations, in which equivalent or lower cutting forces were measured. Eberle et al. [7] demonstrated that the use of picosecond pulses does not result in graphitisation when generating diamond cutting edges, contrary to nanosecond and microsecond pulses. Further shortening the pulse duration, Weikert and Dausinger [8] investigated using a $\tau_{\mathrm{p}}=$ 120 fs to $2 \mathrm{ps}, \lambda=800 \mathrm{~nm}$ and a $\tau_{\mathrm{p}}=15 \mathrm{~ns}, \lambda=1064 \mathrm{~nm}$ laser, the cutting edge generation of monocrystalline diamond (MCD), PCD composite and chemical vapour deposition diamond (CVD-D). The effect of pulse duration, polarisation state, process gas and repetition rate were studied. Joswig et al. [9] used a $\tau_{\mathrm{p}}=150$ to $250 \mathrm{fs}, \lambda=800 \mathrm{~nm}$ laser to generate cutting edges, tool tips and rake face surface structures on CVD-D and single crystal diamond. It was concluded that by using femtosecond pulse durations, no cracking occurs, surface quality is better and high geometrical flexibility is apparent. It is for this reason that only pulse durations in the ultrashort pulse regime, i.e. $\tau_{\mathrm{p}} \leq 10 \mathrm{ps}$, suffice for microcutting tool applications. With respect to the laser processing of cutting tool geometries, only cutting edge generation is heavily investigated. Cutting tools exhibit additional features such as chamfers, tribological structures, chip breakers and various forms of chip guiding geometries which can also be laser processed, by either orthogonal or tangential irradiation. An area where laser technology exhibits superior flexibility is the fabrication of chip breakers. The laser fabrication of chip breakers was previously demonstrated by Dumitru et al. [10] and Miyazawa et al. [11] for tungsten carbide and PCD composites, respectively. This paper will investigate a broad range of topics by covering the direct laser fabrication of cutting edges and chip breakers with feature sizes ranging from $5 \mu \mathrm{m}$ to $1 \mathrm{~mm}$. This is made possible by using picosecond laser pulses operating in the green wavelength and a microscanning system exhibiting a high-numerical aperture. Hence, high-resolution, graphitic carbon-free as well as force- and wear-free fabrication of polycrystalline diamond composites and single diamond grains is demonstrated.

\section{Materials and methods}

A Nd: $\mathrm{YVO}_{4}$ diode pumped MOPA laser system is used which generates pulses at a pulse duration of $\tau_{\mathrm{p}}=10 \mathrm{ps}$. The output wavelength is centred at $\lambda=1064 \mathrm{~nm}$ which is frequency doubled and is linearly polarised. The utilised micro-scanning system described by Gottmann et al. [12] deflects, images

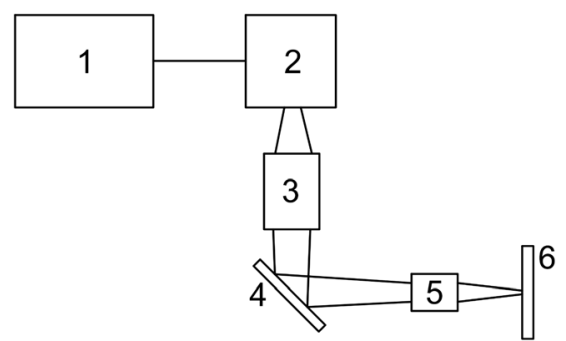

Fig. 1 Experimental setup: (1) laser system, (2) two-axis scanning system with process camera and illumination, (3) imaging optics, (4) bending mirror, (5) microscope objective and (6) workpiece and piezoelectric drives 
Table 1 Summary of laser processing parameters

\begin{tabular}{lll}
\hline Parameter & Value for roughing & Value for finishing \\
\hline Pulse duration, $\tau_{\mathrm{p}}$ & $10 \mathrm{ps}$ & $10 \mathrm{ps}$ \\
Wavelength, $\lambda$ & 532 & 532 \\
Beam quality, $M^{2}$ & 1.1 & 1.1 \\
Fluence, $F$ & 4.1 to $4.7 \mathrm{~J} / \mathrm{cm}^{2}$ & 1.5 to $2 \mathrm{~J} / \mathrm{cm}^{2}$ \\
z-step size, $z_{\text {step }}$ (layer thickness) & $1 \mu \mathrm{m}$ & 100 to $300 \mathrm{~nm}$ \\
Frequency, $f_{\text {rep }}$ & $300 \mathrm{kHz}$ & $300 \mathrm{kHz}$ \\
\hline
\end{tabular}

and focusses the laser beam into a $\times 20,0.4 \mathrm{NA}, f_{\text {eff }}=10 \mathrm{~mm}$ microscope objective. The advantage of this system is its fine focusing ability $\mathrm{D} 4 \sigma<3 \mu \mathrm{m}$, integrated process camera with uniform illumination, as well as its ability to scan over a $\varnothing=$ $1.2 \mathrm{~mm}$ field. Contrary to previous laser-based fabrication techniques where the workpiece is moved relative to a stationary beam, the micro-scanning system deflects the laser beam over a stationary workpiece in the order of $v>100 \mathrm{~mm} / \mathrm{s}$ as opposed to $v=0.08 \mathrm{~mm} / \mathrm{s}$ as demonstrated by Joswig et al. [9]. This enables the scaling of frequency and average power leading to higher material removal rates and hence reduced processing time. The workpiece is positioned and focus-shifted using three-axis linear piezoelectric drives. This form of focus-shifting ensures uniform beam properties throughout processing since its divergence angle and hence spot size does not change. Waveplates in the beam path ensure circular polarisation, and a nozzle around the microscope objective blows clean and dry air coaxially to the processing area. A schematic of the experimental setup is shown in Fig. 1.

Two different types of diamond are investigated: fine grain polycrystalline diamond composites sintered on a tungsten carbide substrate and single diamond grains. The PCD composites (Diamond Innovations Compax 1600) exhibit a mean grain size of $4 \mu \mathrm{m}$ and is composed of $90 \% \mathrm{vol}$ diamond and $10 \%$ vol cobalt. The single diamond grains (Element Six SDB1125) are classified as D426 exhibiting a size ranging from 355 to $425 \mu \mathrm{m}$ and a cubo-octahedral shape. Two processing windows are determined: finishing which achieves the highest ablation quality and roughing which achieves a high ablation rate. The laser parameters are valid both for processing of fine grain PCD composites and diamond grains. The laser parameters are given in Table 1.

The step size of the $z$-axis used for focus-shifting discretises the ablated volume and varies according to the ablated geometry. For geometries exhibiting steep angles, a large z-step size is implemented and vice versa in an effort to minimise discretisation errors. Discretisation error is defined as the deviation from the ideal geometry due to poor approximation. The main processing strategy, depending on the geometry, follows either tangential or orthogonal irradiation aided by $\mathrm{CAD} / \mathrm{CAM}$ software. However, cutting edges and similar geometries follow the principles made clear by Dold [13]. In both cases, the concept of 2.5D laser ablation is implemented to generate 3D geometries. High-resolution imaging is made possible through scanning electron microscopy using a secondary electron (SE) detector. Cutting edges are initially gold sputter coated and then measured using a focus variation $3 \mathrm{D}$ optical microscope and a $0.55 \mathrm{NA}, \times 50$ objective. Raman measurements use a confocal Raman microscope and a $0.80 \mathrm{NA}, \times 100$ objective at an excitation wavelength of $\lambda=532 \mathrm{~nm}$.

Fig. 2 Thin-walled structures similarly found in heat sink applications

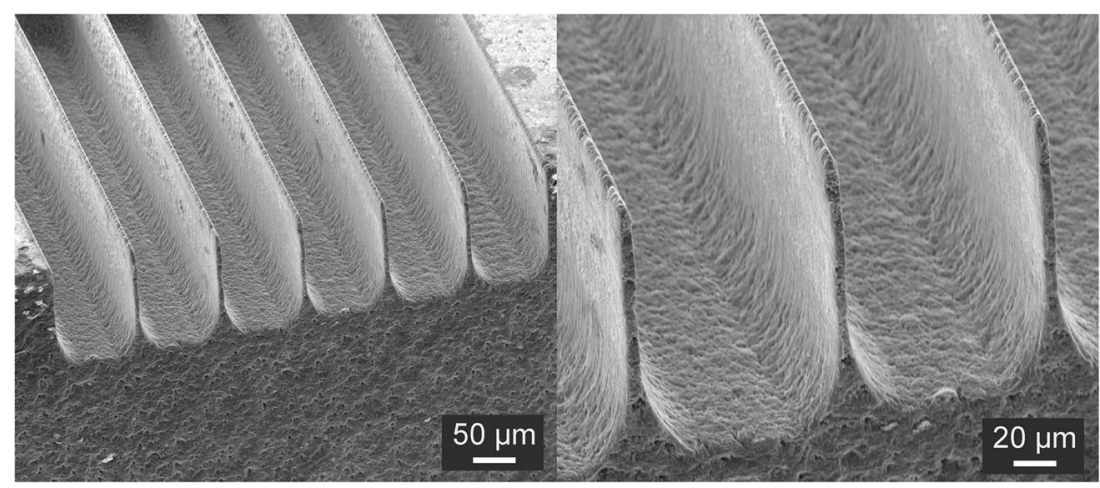


Fig. 3 Periodic polygon structures similarly found in grinding pads

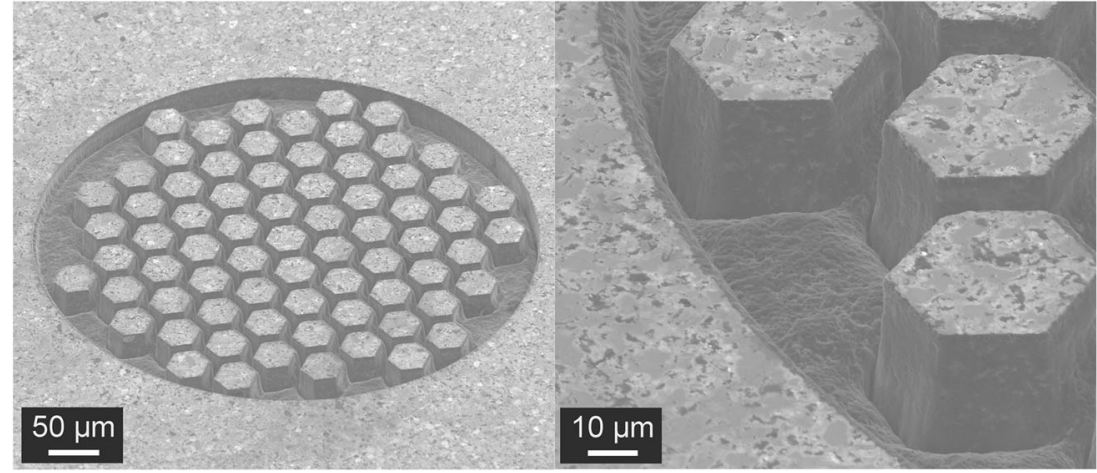

\section{Results and discussion}

\subsection{Periodic structures}

To demonstrate the capability of the micro-scanning system, two types of periodic structures are generated where both fineness and quality are emphasised. The ribbed structures in Fig. 2 are processed similar to a cutting edge at a $0^{\circ}$ inclination angle. The geometry exhibits a rib thickness of $5 \mu \mathrm{m}$, a depth of $120 \mu \mathrm{m}$ and an inter rib spacing of $100 \mu \mathrm{m}$. When comparing the rib thickness with the mean grain size of the PCD composite, structural integrity is apparent indicating that processing is carried out with negligible thermal influences and independent of material composition. Potential applications of such structures is envisioned as heat sinks, especially for microelectronics.

The polygon structures in Fig. 3 exhibit an apothem of $20 \mu \mathrm{m}$ and a depth $<10 \mu \mathrm{m}$ with a diameter of $\varnothing=500 \mu \mathrm{m}$. Upon closer inspection, the walls of the polygon structure seem polished and nicely show the interface between cobalt in white and the diamond in black. Such polishing quality of diamond is often associated with ion beam technologies. Potential applications of such structures are envisioned as microgrinding pads.

Since PCD composites are electrically conductive, further applications can encompass EDM electrodes. The generated periodic geometries are parallel to the optical axis of the laser beam, i.e. no angular geometries are present, implying tangential irradiation. Hence, the laser parameters for roughing is chosen since high ablation rates can be achieved while still maintaining narrow form tolerances.

\subsection{Cutting edge generation}

Diamond grains are often stochastically bonded to dressing or grinding wheels. Dold [13] demonstrates the laser processing of electroplated stochastically bonded dressing wheels so as to generate a quasi-engineered grinding tool. Engineered grinding tools (EGT) eliminate the stochastic nature of grinding wheels leading to high removal rates and defined grinding behaviours which can be tuned as noted by Pinto et al. [14]. A further improvement to EGT is presented in this paper by demonstrating the ability to shape single diamond grains as shown in Fig. 4. A typical unprocessed diamond grain is shown in Fig. 4 (left) followed by a laser processed diamond grain in Fig. 4 (right). Both a defined clearance face and rake face is fabricated. In doing so, the volume of the diamond grain is reduced by approximately one half. In general, to achieve a sharp cutting edge, the rake face is initially processed starting from the cutting edge down to a defined depth. This is followed by the clearance face which is processed starting from a defined depth towards the cutting edge.

Fig. 4 Laser shaping of a diamond grain for EGT applications

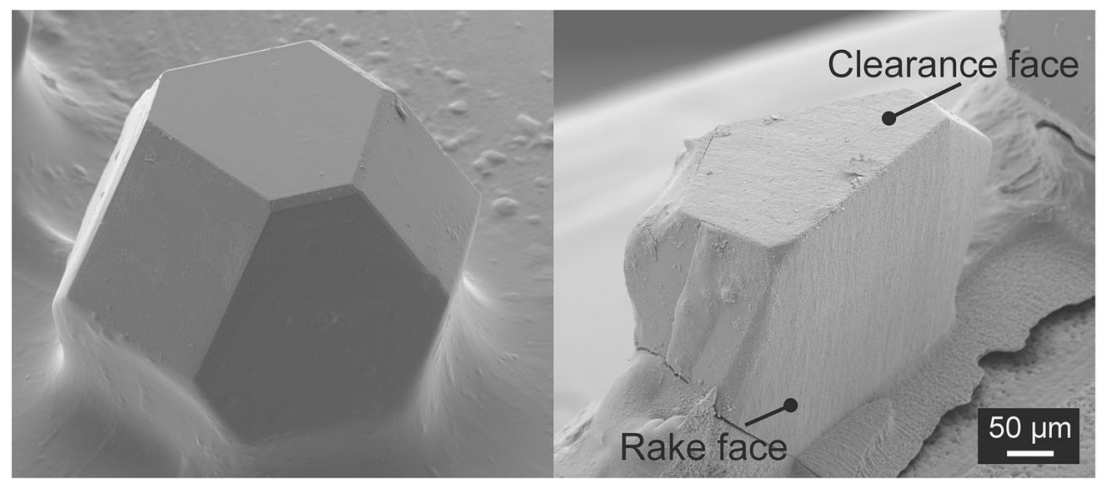


Fig. 5 Profile (left) and surface topography (right) of the measured cutting edge on a D426 diamond grain
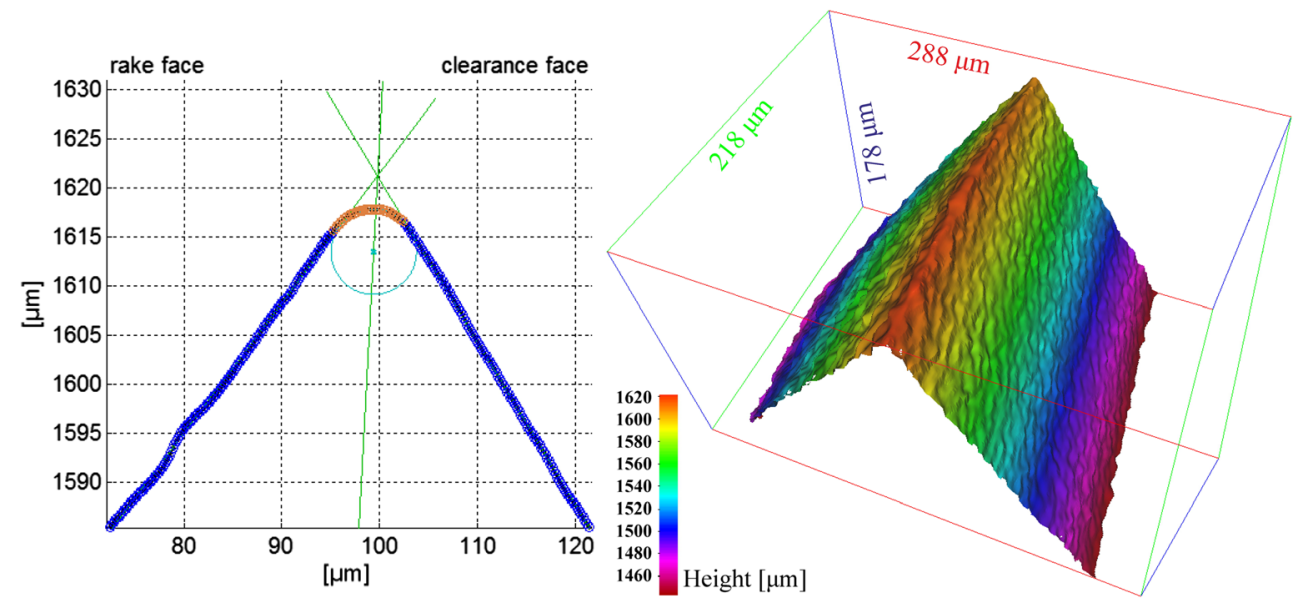

The cutting edge radius and wedge angle of the laser processed grain are measured and determined according to the approach outlined by Wyen and Wegener [15] and Heneriches et al. [16]. An ideal $r_{\text {edge }}=4.4 \mu \mathrm{m}$ and a wedge angle of $\beta=68^{\circ}$ are determined. A typical averaged profile of the cutting edge is shown in Fig. 5. What can be especially noticed is that there are no waterfall effects and the profile shows near symmetry.

According to Yuan et al. [17], the cutting edge radius has a considerable influence on the machined surface integrity with respect to surface roughness, micro-hardness, residual stress and dislocation density. For this reason, the cutting edge radius can be modified by modulating the laser energy at the cutting edge. This implies that the level of laser energy at the tails of the scanned Gaussian laser beam can be used to influence the radius. The approach of tangential irradiation and the laser parameters for roughing are used to generate the cutting edge geometries.

\subsection{Chip breakers}

The generation of chips in a machining process is an inherent characteristic, present both in macro- and micromachining. Chip morphology can be categorised as continuous, continuous with built-up edge, discontinuous and shear localised. Chip breaker geometries are designed to control the growth and curl or radius of curvature of the outgoing chip in order to induce high strains at the outer surface of the chip. According to Choi and Lee [18], a chip will break if the chip tensile strain is greater than the chip fracture (ultimate tensile) strain, such that tensile strain is a function of chip thickness and chip curl radius. Depending on the chip breaker design or through the addition of chip guiding geometries, the broken chip is subsequently conveyed away from the workpiece. Chip breakers are often designed and optimised according to simulation studies. Parameters identified by Jawahir and Fang [19] which influence its design include machined material, cutting edge radius, depth of cut, feed rate, cutting speed and inclination angle. Figure 6 shows a chip breaker found in an indexable insert used in turning operations. It exhibits a tip-to-tip width of $500 \mu \mathrm{m}$, a depth of $28 \mu \mathrm{m}$ and a chip breaker rake angle of $12^{\circ}$. The design of this chip breaker is considered a $3 \mathrm{D}$ chip breaker.

Figure 7 shows a chip breaker found in a rotationally symmetric cutting tool, e.g. an end mill used in milling operations. It exhibits a length of $1 \mathrm{~mm}$, a width of $100 \mu \mathrm{m}$ and a depth of
Fig. 6 Chip breaker geometry for an indexable insert

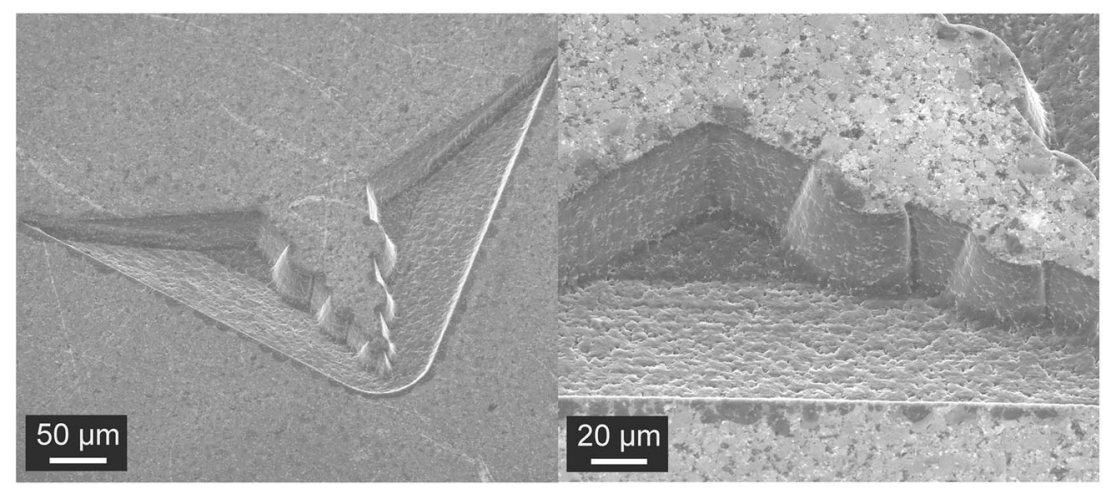


Fig. 7 Chip breaker geometry for an end mill

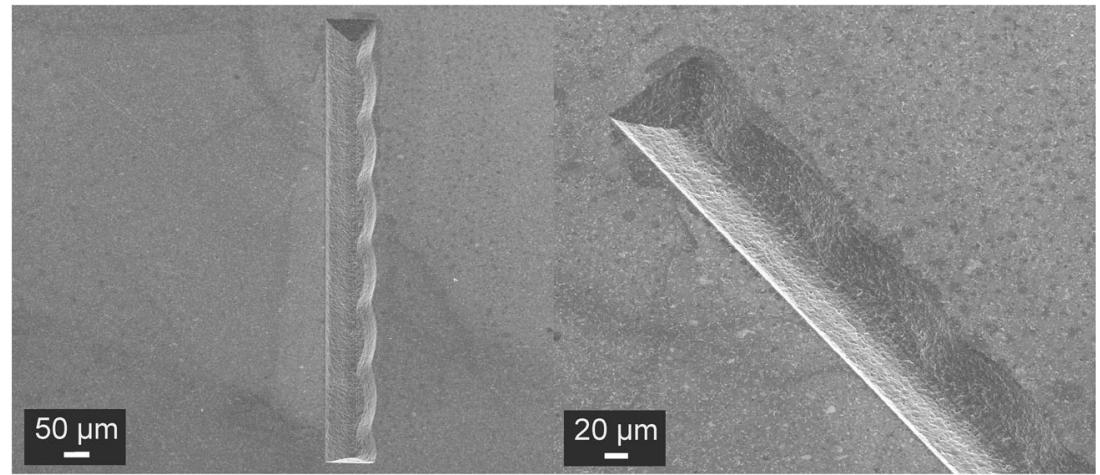

$24 \mu \mathrm{m}$. The design of this chip breaker follows closely to that of a groove-type chip breaker.

The final type of chip breaker, shown in Fig. 8, is found in indexable inserts, often made of cemented carbide. It exhibits a symmetric side length of $750 \mu \mathrm{m}$, a depth of $30 \mu \mathrm{m}$ and a chip breaker rake angle of $22^{\circ}$. This type of geometry demonstrates the resolution limits of the micro-scanning system in which unlike all other geometries, minimal discretisation error is apparent. This can be solved by reducing the z-step size and the use of an objective with a higher numerical aperture.

Due to geometric feature intricacies and the chip breaker rake angle, the approach of orthogonal irradiation and the laser parameters for finishing are used to generate the chip breaker geometries.

\subsection{Process improvement}

The 2.5D laser ablation process follows a similar but wellestablished concept used in selective laser melting (SLM) or selective laser sintering (SLS). As opposed to building up a 3D structure in a layer-wise fashion according to the geometry's cross-section, the opposite approach is taken for 3D laser ablation, i.e. layer-wise removal. The limitation of both processes is that the layer thickness defines the axial resolution of the resulting geometry as well as processing time. The dilemma is that resolution and processing time exhibit an inverse relationship. Optimising either for axial resolution or for processing time defines the severity of discretisation error. To mitigate this shortfall, a sequential two-step process is proposed. Initially, roughing of a large material allowance occurs at high fluences $\left(F>4 \mathrm{~J} / \mathrm{cm}^{2}\right)$ and large layer thicknesses. This is followed by finishing of the outer contour of the geometry at low fluences or fluences near ablation threshold $\left(F \leq 2 \mathrm{~J} / \mathrm{cm}^{2}\right)$ and small layer thicknesses. Laser parameters are altered at each step according to Table 1. This two-step concept along with two different approaches are illustrated in Fig. 9.

During roughing, the first approach in Fig. 9 (left) ablates less volume compared to the second approach in Fig. 9 (right). The advantage though is that one can utilise far higher fluences and larger layer thicknesses since discretisation errors are of no issue. Alternatively, the approach given in Fig. 9 (right) results in less material allowance which needs to be ablated during finishing. For proof of concept, the first approach is tested and results are shown in Fig. 10.

The ablation volume of the given example for roughing and for finishing amounts to $1.4 \times 10^{-3}$ and $0.9 \times 10^{-3} \mathrm{~mm}^{3}$, respectively, leading to a total ablation volume of $2.1 \times$ $10^{-3} \mathrm{~mm}^{3}$. An overlap of the two ablation volumes is necessary to consider mismatch of ablation rates and results in seamless transition from roughing to finishing. The majority of volume is removed during the roughing step which requires

Fig. 8 Full geometry of a turning indexable insert

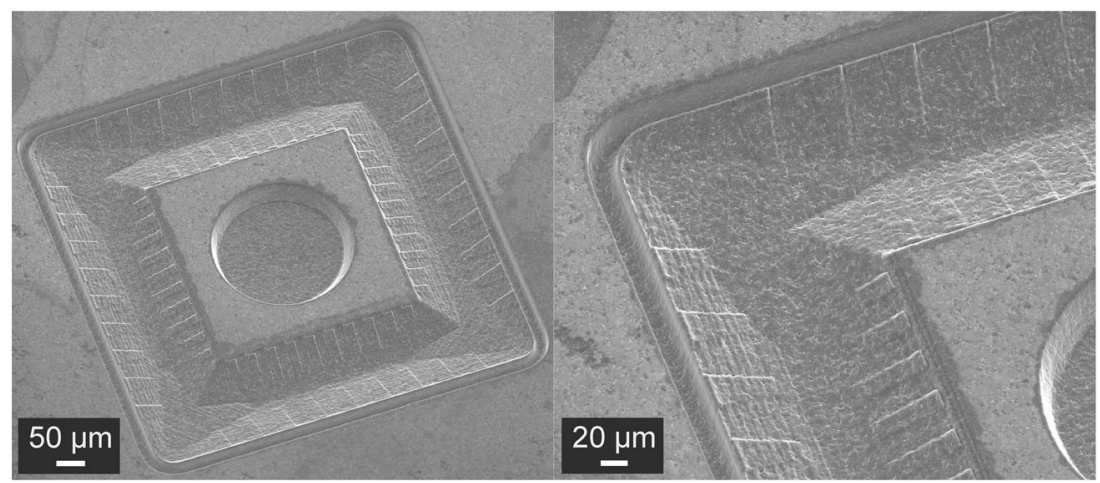


Fig. 9 Two different approaches proposed to optimise $2.5 \mathrm{D}$ laser ablation through a two-step process

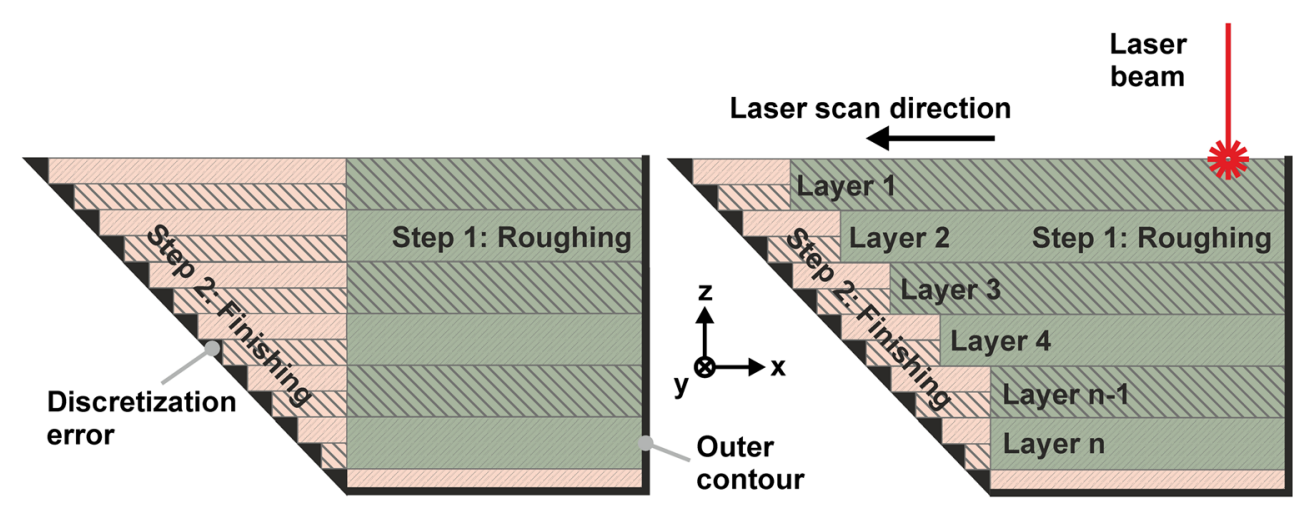

approximately $t_{\mathrm{r}} \approx 1.3 \mathrm{~min}$. This is followed by finishing of the remaining volume which requires $t_{\mathrm{f}} \approx 25 \mathrm{~min}$. It must be considered however that scanner and laser delays and more importantly the hatch geometry play an important role within this topic and can either worsen or improve the processing time when using the sequential two-step concept.

\subsection{Quality}

An evaluation of surface roughness during tangential and orthogonal irradiation is previously covered by Eberle et al. [20]. During tangential irradiation, a polished surface can be generated as shown in Fig. 11 (top right). Here, the material composition between PCD and cobalt can be easily differentiated and surface roughness lies in the order of $R_{\mathrm{a}}<90 \mathrm{~nm}$. During orthogonal irradiation, the ablation threshold difference between PCD and cobalt significantly influences the resulting quality. Since the ablation threshold of diamond and cobalt in the picosecond time regime is not found in literature, the ablation threshold in the nanosecond time regime is given as a reference. According to Li et al. [21] and Ageev et al. [22], the multipulse ablation threshold of cobalt is $F_{\mathrm{th}}=$ $0.6 \mathrm{~J} / \mathrm{cm}^{2}$ whereas the multipulse ablation threshold of diamond is $F_{\text {th }}=1 \mathrm{~J} / \mathrm{cm}^{2}$, respectively. Both authors present values using a $\mathrm{XeCl}$ excimer laser at $\tau_{\mathrm{p}}=20 \mathrm{~ns}, \lambda=308 \mathrm{~nm}$. Due to this threshold difference, the presence of cobalt is diminished revealing pores as shown in Fig. 11 (bottom right), since cobalt is initially ablated, followed by diamond. Surface roughness after orthogonal irradiation, unlike tangential irradiation, and particularly for multi-component materials is highly dependent on laser fluence where $R_{\mathrm{a}}<200 \mathrm{~nm}$ when $F<8.5 \mathrm{~J} / \mathrm{cm}^{2}$.

Figure 11 is a magnified inset of a feature from Fig. 10. It is generally understood, as stated by Kuznetsov and Butenko [23], that diamond graphitises when it reaches a temperature of $T_{\mathrm{g}} \gtrsim 700{ }^{\circ} \mathrm{C}$ at atmospheric pressure and given the presence of oxygen. Furthermore according to Corbari et al. [24], electron-phonon coupling for silica glass (insulating electronic band structure similar to diamond) commences when $\tau_{\mathrm{p}}=1 \mathrm{ps}$ and continues in the order of picoseconds followed by ablation. The energy conducted during electron-phonon relaxation although occurring over a very short time scale can lead to diamond graphitisation, especially since the graphitisation onset temperature is very low. For these two reasons, it can be argued that picosecond ablation of diamond is aided either by the graphitisation process or by dielectric breakdown. Though this topic lies outside the scope of this paper, using ultrashort pulse durations and by using appropriate laser parameters as well as processing strategies, graphitisation can be kept to a minimum or even avoided as shown in the Raman spectra in Fig. 12. The initial conditions of the unprocessed diamond is plotted as well as after various laser processing conditions and at different positions.

Typically, graphitic carbons exhibit a peak in the D-band at $\sim 1360 \mathrm{~cm}^{-1}$, G-band at $\sim 1580 \mathrm{~cm}^{-1}$ and 2D-band at $\sim 2700 \mathrm{~cm}^{-1}$, while crystallographic diamond only exhibits a peak at $1332 \mathrm{~cm}^{-1}$. Depending on the type of graphitic carbon, the peaks at each respective band can shift considerably and even be non-existent depending on the graphitic carbon crystallinity. Referring to the Raman spectrum of the diamond
Fig. 10 Evolution of the two-step approach: after roughing (left), intermediately through finishing (middle) and the finished geometry (right)

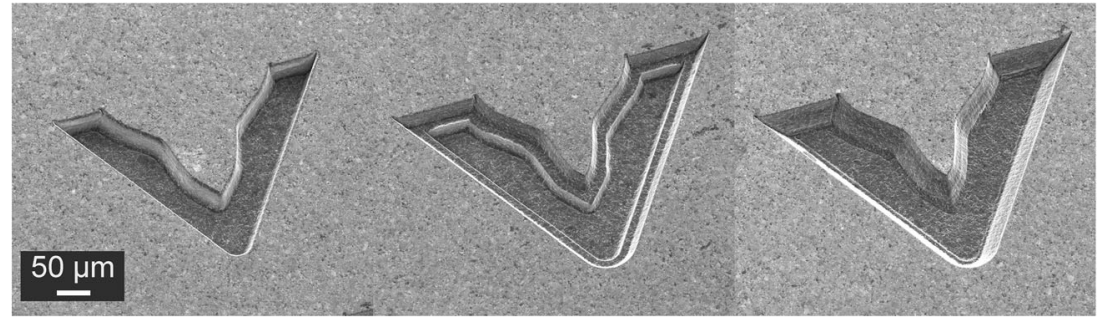


Fig. 11 Ablation quality: tangentially irradiated surface (top right) and orthogonally irradiated surface (bottom right)
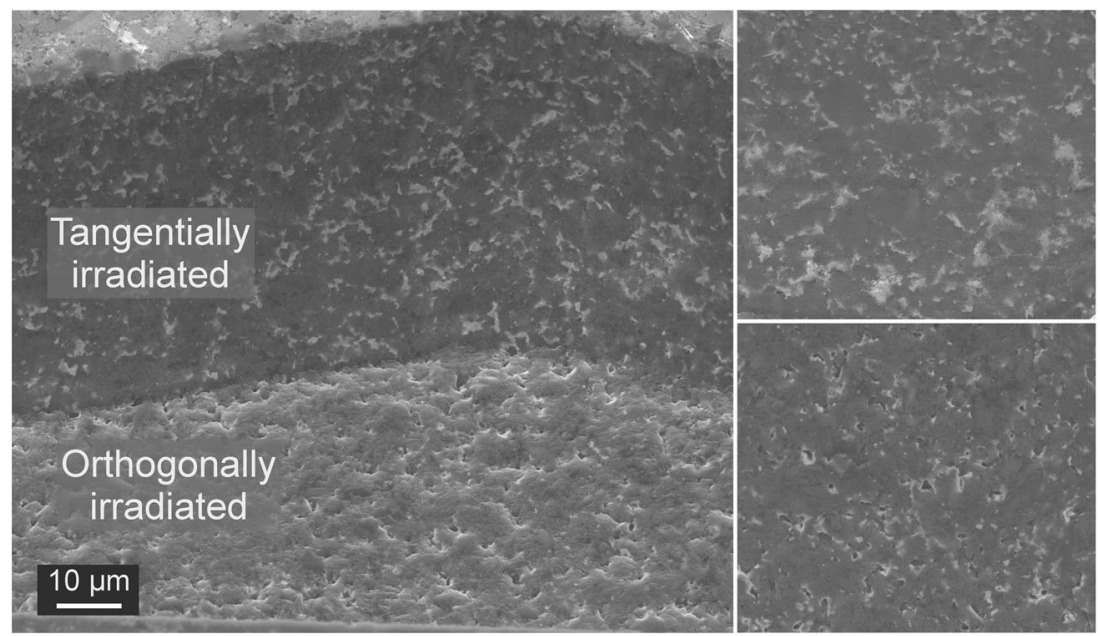

grain, after laser processing the clearance face (black curve), there exists a peak in the D-band and G-band, with strong broadening indicating graphitic carbon with disorder, i.e. a mixture of $\mathrm{sp}^{2}$ (graphite-like) and $\mathrm{sp}^{3}$ (diamond-like) phases. Since there is no second-order peak, this indicates a transformation towards amorphous carbon. These characteristics can be found in glassy carbon. The spectrum for the rake face (blue curve) exhibits only crystallographic diamond, i.e. a peak at $1332 \mathrm{~cm}^{-1}$. The reason for the difference between the two faces is that during clearance face generation, the laser beam is only in partial contact with the material and the rest in air, while full material contact occurs during rake face generation. For this reason, the thermal load on the clearance face is higher. Solutions to avoid graphitisation is the condition of full material contact or by performing a finishing step at fluences near or at the ablation threshold. When inspecting the Raman spectra for PCD composites, the signal is noisier and the signal increasingly broadens to higher intensities compared to the diamond grain. This is attributed to fluorescence effects from the cobalt binder which arises from intense resonance effects, or rather over-excitation of metallic (cobalt) atoms leading to a strong intensity signal. After tangential irradiation (green curve), only crystallographic diamond is present. After orthogonal irradiation (magenta curve), the Raman spectrum exhibits a strong peak at $1332 \mathrm{~cm}^{-1}$, while there is evidence of a broad and shallow peak in the G-band, indicating a high proportion of $\mathrm{sp}^{3}$ bonds with a slight onset of graphitisation. Residual graphitic carbon on laser processed geometries can arise from two possibilities: either by heat conduction or by recast of the vaporised diamond in the form of amorphous carbon. In the first case, it is expected that the graphitic carbon layer will be smooth and uniform while the latter case will show high roughness and flaws. The bonding behaviour of graphitic carbon on diamond is weak because of the inherent properties of graphitic carbon and because of the presence of two different material phases. Hence, graphitic carbon can be
Fig. 12 Raman spectroscopy measurements on polycrystalline diamond composites and diamond grains

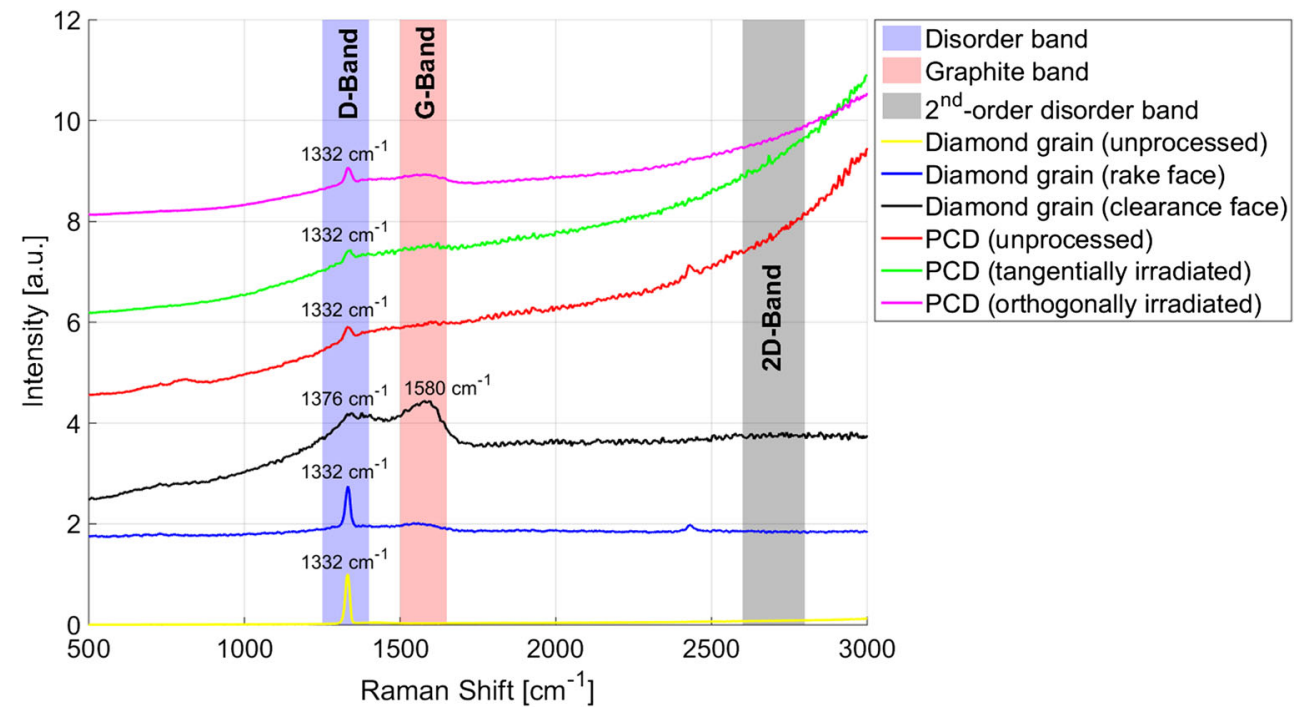


easily removed when implemented in a machining process and can be removed by acid treatments or by thermalchemical refinement as demonstrated by Zong et al. [25].

\section{Conclusions}

A direct laser fabrication technique is demonstrated which is able to generate, without any process forces or wear, fine geometries applicable to micro-cutting tools. Geometries include periodic structures, cutting edges and chip breakers made of polycrystalline diamond composites and single diamond grains, whereby errors caused by volume discretisation is negligible. Differentiation between orthogonal and tangential irradiation is demonstrated such that, regardless of irradiation orientation, graphitisation of diamond can be avoided. To accelerate the $2.5 \mathrm{D}$ laser ablation process, a sequential twostep approach of roughing and finishing is verified. The kinematic setup of the workpiece positioner can be extended to five axes, and the objective can be upgraded with a higher numerical aperture, allowing for 3D laser ablation and higher geometric feature resolution, further enhancing the capabilities of the micro-scanning system.

Acknowledgments The authors would like to thank for the use of equipment by the Electron Microscopy Center and the Micro- and Nanosystems Group of ETH Zurich as well as for the financial support from the Swiss Commission for Innovation and Technologies.

\section{References}

1. Klotzbach A, Hauser M, Beyer E (2011) Laser cutting of carbon fiber reinforced polymers using highly brilliant laser beam sources. Phys Procedia 12:572-577. doi:10.1016/j.phpro.2011.03.072

2. Ancona A, Röser F, Rademaker K, Limpert J, Nolte S, Tünnermann A (2008) High speed laser drilling of metals using a high repetition rate, high average power ultrafast fiber CPA system. Opt Express 16:8958-8968. doi:10.1364/OE.16.008958

3. Huo D, Cheng K (2013) Overview of micro cutting. In: Cheng K, Huo D (eds) Micro-cutting: fundamentals and applications, 1 st edn. Wiley, West Sussex, pp 10-14

4. Harrison P, Henry M, Brownell M (2006) Laser processing of polycrystalline diamond, tungsten carbide and a related composite material. J Laser Appl 18:117-126. doi:10.2351/1.2164472

5. Everson C, Molian P (2009) Fabrication of polycrystalline diamond microtool using a Q-switched Nd:YAG laser. Int J Adv Manuf Technol 45:521-530. doi:10.1007/s00170-009-1999-6

6. Dold C, Henerichs M, Gilgen P, Wegener K (2013) Laser processing of coarse grain polycrystalline diamond (PCD) cutting tool inserts using picosecond laser pulses. Phys Procedia 41:610-616. doi:10.1016/j.phpro.2013.03.123

7. Eberle G, Jefimovs K, Wegener K (2014) Characterisation of thermal influences after laser processing polycrystalline diamond composites using long to ultrashort pulse durations. Precis Eng 39:1624. doi:10.1016/j.precisioneng.2014.06.008
8. Weikert M, Dausinger F (2004) Cutting of diamond. In: Dausinger F, Lubatschowski H, Lichtner F (eds) Femtosecond technology for technical and medical applications. Springer, Berlin/Heidelberg, pp 155-165

9. Joswig A, Risse S, Eberhardt R, Tünnermann A (2010) Laser generated and structured prototypes of diamond tool tips for microoptics fabrication. In: 25th Annual Meeting of the American Society for Precision Engineering: Proceedings 31, Atlanta, USA, pp 53-56

10. Dumitru G, Lüscher B, Krack M, Bruneau S, Hermann J, Gerbig Y (2005) Laser processing of hardmetals: physical basics and applications. Int J Refract Met Hard 23:278-286. doi:10.1016/j.ijrmhm. 2005.04.020

11. Miyazawa H, Takeuchi S, Miyake S, Murakawa M (1996) Sintered diamond cutting inserts with chip breaker prepared by laser technique. Surf Coat Technol 86-87:797-802. doi:10.1016/S02578972(96)03068-X

12. Gottmann J, Horstmann-Jungemann M, Hermans M, Beckmann D (2009) High speed and high precision fs-laser writing using a scanner with large numerical aperture. J Laser Micro/Nanoeng 4:192196. doi: $10.2961 /$ jlmn.2009.03.0009

13. Dold C (2013) Picosecond laser processing of diamond cutting edges. VDI-Verlag, Düsseldorf

14. Pinto F, Vargas G, Wegener K (2008) Simulation for optimizing grain pattern on engineered grinding tools. CIRP Ann Manuf Technol 57:353-356. doi:10.1016/j.cirp.2008.03.069

15. Wyen C, Wegener K (2010) Influence of cutting edge radius on cutting forces in machining titanium. CIRP Ann Manuf Technol 59:93-96. doi:10.1016/j.cirp.2010.03.056

16. Henerichs M, Voss R, Kuster F, Wegener K (2014) Charakterisierung der Schneidkantengeometrie für Standzeitoptimierung beim Bohren von CFK. Diam Bus 4:42-47

17. Yuan Z, Zhou M, Dong S (1996) Effect of diamond tool sharpness on minimum cutting thickness and cutting surface integrity in ultraprecision machining. J Mater Process Technol 62:327-330. doi: 10.1016/S0924-0136(96)02429-6

18. Choi J, Lee S (2001) Efficient chip breaker design by predicting the chip breaking performance. Int J Adv Manuf Technol 17:489-497. doi:10.1007/PL00003947

19. Jawahir I, Fang X (1995) A knowledge-based approach for designing effective grooved chip breakers - 2D and 3D chip flow, chip curl and chip breaking. Int J Adv Manuf Technol 10:225-239. doi: 10.1007/BF01186875

20. Eberle G, Dold C, Wegener K (2015) Picosecond laser fabrication of micro cutting tool geometries on polycrystalline diamond composites using a high-numerical aperture micro scanning system. Proc SPIE:9351. doi: 10.1117/12.2075420

21. Li T, Lou Q, Dong J, Wei Y, Liu J (2001) Ablation of cobalt with pulsed UV laser radiation. Appl Surf Sci 172:356-365. doi:10. 1016/S0169-4332(00)00883-7

22. Ageev P, Builov L, Konov I, Kuzmichev V, Pimenov M (1988) Interaction of laser radiation with diamond films. Dokl Akad Nauk SSSR 303:598-601

23. Kuznetsov V, Butenko Y (2012) Diamond phase transitions at nanoscale. In: Shenderova O, Gruen D (eds) Ultrananocrystalline diamond, 2nd edn. William Andrew, Waltham/Oxford, pp 181-244

24. Corbari C, Champion A, Gecevicius M, Beresna M, Bellouard Y, Kazansky P (2013) Femtosecond versus picosecond laser machining of nano-gratings and micro-channels in silica glass. Opt Express 21:3946-3958. doi:10.1364/OE.21.003946

25. Zong W, Zhang J, Liu Y, Sun T (2014) Achieving ultra-hard surface of mechanically polished diamond crystal by thermochemical refinement. Appl Surf Sci 316:617-624. doi:10. 1016/j.apsusc.2014.08.057 